This item was submitted to Loughborough's Research Repository by the author.

Items in Figshare are protected by copyright, with all rights reserved, unless otherwise indicated.

\title{
Top fuel dragster powertrain modelling
}

PLEASE CITE THE PUBLISHED VERSION

http://www.elecpubs.sae.org/NXT/gateway.dll?f=templates\&fn=default.htm

PUBLISHER

(C) SAE International

VERSION

VoR (Version of Record)

LICENCE

CC BY-NC-ND 4.0

REPOSITORY RECORD

Stone, Thomas C., Sebastian Visser, and Matt C. Best. 2011. "Top Fuel Dragster Powertrain Modelling”. figshare. https://hdl.handle.net/2134/8397. 
This item was submitted to Loughborough's Institutional Repository (https://dspace.lboro.ac.uk/) by the author and is made available under the following Creative Commons Licence conditions.

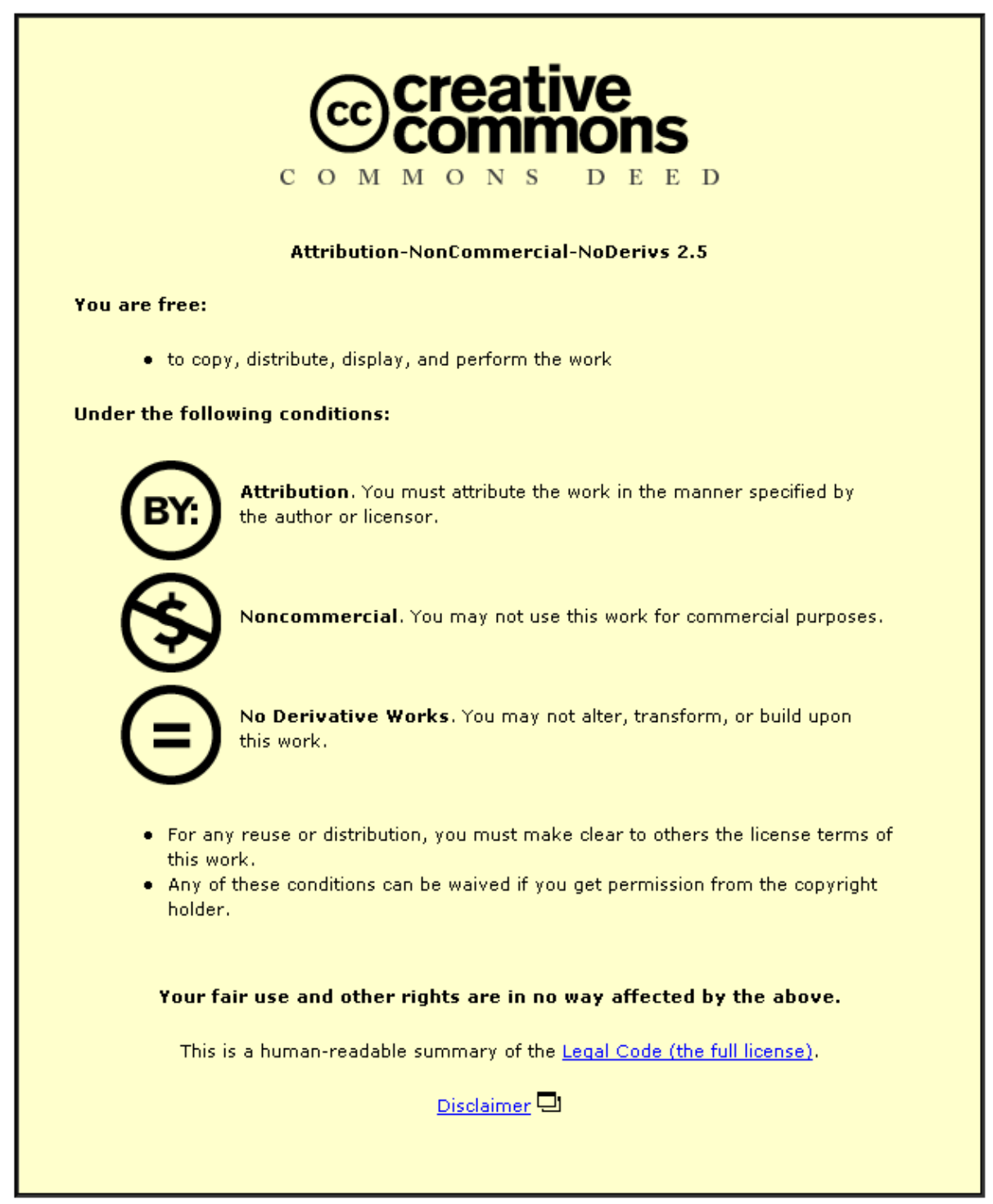

For the full text of this licence, please go to: http://creativecommons.org/licenses/by-nc-nd/2.5/ 


\section{Top Fuel Dragster Powertrain Modelling}

Thomas C. Stone and Sebastian Visser

Lex Joon Racing

Matthew C. Best

Loughborough University 
The Engineering Meetings Board has approved this paper for publication. It has successfully completed SAE's peer review process under the supervision of the session organizer. This process requires a minimum of three (3) reviews by industry experts.

All rights reserved. No part of this publication may be reproduced, stored in a retrieval system, or transmitted, in any form or by any means, electronic, mechanical, photocopying, recording, or otherwise, without the prior written permission of SAE.

For permission and licensing requests contact:

SAE Permissions
400 Commonwealth Drive
Warrendale, PA 15096-0001-USA
Email: permissions@ @ sae.org
Tel: $\quad 724-772-4028$
Fax: $\quad 724-776-3036$

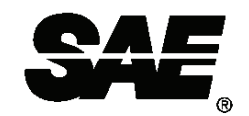

Global Mobility Database ${ }^{\circledR}$

All SAE papers, standards, and selected books are abstracted and indexed in the Global Mobility Database.

For multiple print copies contact:

SAE Customer Service

Tel: $\quad$ 877-606-7323 (inside USA and Canada)

Tel: $\quad$ 724-776-4970 (outside USA)

Fax: $\quad$ 724-776-0790

Email: CustomerService@sae.org

\section{ISSN 0148-7191}

\section{Copyright $\odot 2008$ SAE International}

Positions and opinions advanced in this paper are those of the author(s) and not necessarily those of SAE. The author is solely responsible for the content of the paper. A process is available by which discussions will be printed with the paper if it is published in SAE Transactions.

Persons wishing to submit papers to be considered for presentation or publication by SAE should send the manuscript or a 300 word abstract to Secretary, Engineering Meetings Board, SAE. 


\title{
Top Fuel Dragster Powertrain Modelling
}

\author{
Thomas C. Stone and Sebastian Visser \\ Lex Joon Racing \\ Matthew C. Best \\ Loughborough University
}

Copyright @ 2008 SAE International

\begin{abstract}
Here we consider the construction of a model in the matlab/simulink environment that describes the dynamic processes occurring within the driveline of a Top Fuel Dragster. Particular emphasis is placed upon development of the model for aspects of powertrain performance that are unique to operation of a dragster. This includes clutch operation, tyre modelling including squat and growth and vertical load variation. The model predictions are compared to vehicle test data, and although predictions are not accurate clear similarities are seen. Hence a good basis for simulating the dragster has been produced but significant parameter tuning work is still required. The paper considers future enhancements to the model and methods for identifying model parameters, in order to further improve accuracy.
\end{abstract}

\section{INTRODUCTION}

The top fuel dragster is designed solely for the purpose of covering a standing quarter mile as quickly as possible. Within the top fuel racing class, dragsters are required to run on a mixture of nitro methane and methanol. The dragsters have V8 engines of around 500 cubic inches and can produce in excess of $8000 \mathrm{HP}$. The Lex Joon Racing Top Fuel dragster has a wheelbase of 7.62 metres and weighs $1023 \mathrm{~kg}$ including the driver. The dragster is capable of around $4 \mathrm{~g}$ acceleration during launch from the start line and can reach speeds in excess of $300 \mathrm{MPH}$. Times under 5 seconds are regularly recorded for the quarter mile.

The dragster has no gearbox. The engine outputs to a centrifugal multi-plate clutch, which in turn outputs to a driveshaft that is connected to a reverser (only used for pre-run burnout). After the reverser the driveline continues to the final drive. This final drive directs the torque to the single rear axle which is connected to the wheel hubs.

A standard compliant driveline model is used as the basis for the model. The paper then concentrates on the components which are unique to the top fuel dragster.
The first of these is the clutch model; a physical model has been developed to describe the complex motion of the clutch release mechanism. All tuning parameters available in the actual clutch assembly are included, along with appropriate, if nominal temperature and clutch pack wear considerations.

A further key development area is in the driven wheels. These have very large, under-inflated tyres which provide enormous traction, but which also experience significant compliance effects both radially, and in torsion. The model tackles these by combining a semi-empirical (Pacejka) formula with some appropriate compliant elements, and with proper consideration given to vertical loads applied by aerodynamics and load transfer.

\section{POWERTRAIN MODEL OVERVIEW}

Figure 1 shows a schematic of the top fuel dragster powertrain model. The engine torque curve provides torque to the system such that engine torque is a function of time, spark timing, throttle, and flywheel (engine) angular velocity.

The amount that changes in spark timing affect the torque value is modelled according to typical values as presented by Heywood [4]. Retardation in ignition timing to $78 \%$ of MBT timing yields a torque multiplication factor of 0.975 . Retardation to $44 \%$ of MBT timing yields a torque multiplication factor of 0.9 ; the model interpolates between these points. In addition the model will interpolate for timings more advanced of MBT in a symmetrical manner.

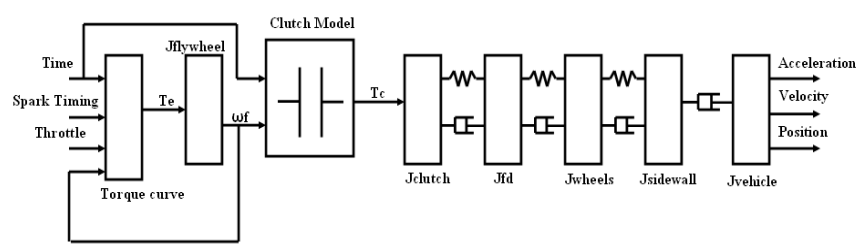

Figure 1: Schematic of Powertrain Model 
The remainder of the system is modelled as a series of rotating masses and a final translational mass connected through spring / damper or clutch models. The clutch connects the flywheel and clutch pack inertias, where torque transmitted is a function of input engine speed and time, in addition to clutch settings defined in the initialisation file.

The tyre sidewall is modelled as a separate rotating mass to the wheel since displacement in the connecting spring-damper equates to sidewall compliance. The connection between the sidewall and the vehicle mass is modelled as a damper, since this accurately represents tyre behaviour.

\section{CLUTCH OPERATION INFORMATION}

The clutch release mechanism is illustrated in Figure 2. The clutch pack comprises 5 discs separated by four floaters. At the rear of the pack are a pressure plate and an external finger plate which are mounted to the flywheel. The external finger plate has 18 metal 'fingers' fitted radially. Due to the rotation of the finger plate these experience centrifugal force which acts to make them rotate around their pivot towards a horizontal position.

Depending on its position, each finger is constrained by, and hence exerts pressure on, either the canon and release bearing, or the clutch pack pressure plate.

The fingers have varying geometries so that, as the bearing moves backwards, they are released on to the pressure plate sequentially. They also have adjustable weights fitted to them.

The hydraulic flow at the rear of the canon is controlled by 6 pneumatic timers which are set off by the driver via a button at the end of the throttle pedal travel. The timers operate valves (or jets) which return fluid from the hydraulic chamber of the canon to an accumulator. Clutch release is thus tuned by finger geometry, adjustable weights, and the six jet timings, to establish a pre-defined sequential activation of the clutch.

Of the 18 fingers, six are primary fingers, with greater mass, and the remaining 12 are secondary. The canon is usually pre-charged with fluid such that the release bearing starts in the position where the third secondary finger has not been released. The first 8 fingers thus provide an initial clamp load to the pack. There is also a preload applied to the pressure plate by springs.

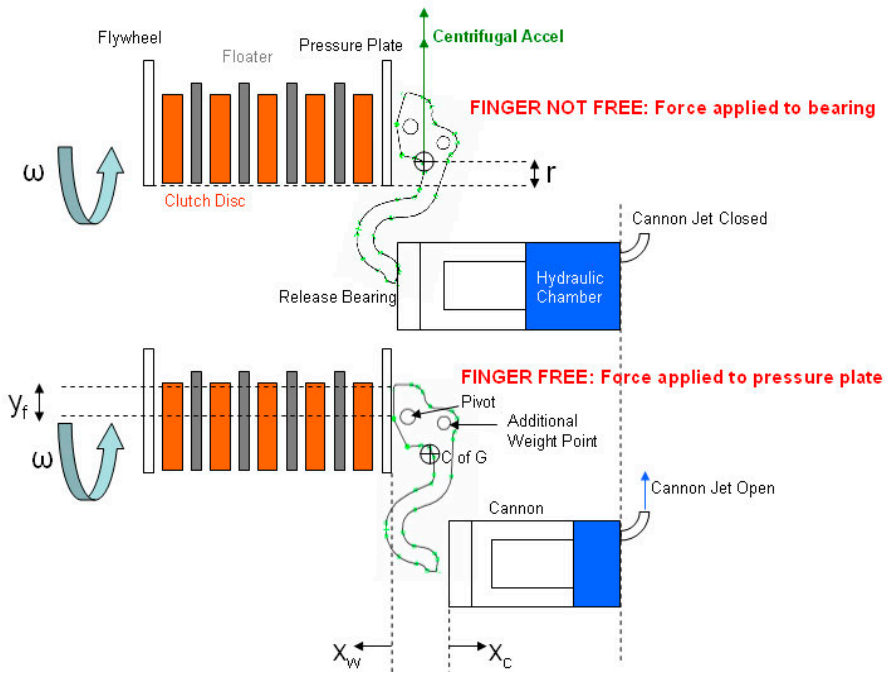

Figure 2: Centrifugal Clutch System Schematic

\section{CLUTCH MODEL DEVELOPMENT}

There are three subsystems within the clutch model. These are:

1. Clamp Load Application

2. Temperature, work and wear

3. Clutch torque calculation

These three systems interact to produce a slipping clutch torque value, which applies when significant difference in speeds exists between the input (flywheel) and the output (clutch pack) angular velocities. An equivalent, closed clutch torque is modelled by a high gain linear damper acting between the two inertias. Then if the slipping torque is less than the closed torque then the clutch is slipping, and if the converse is true, the velocities are closely matched.

The three subsystems are described below.

CLAMP LOAD APPLICATION - This subsystem models the dynamics of the clutch fingers and the cannon system. Figure 2 illustrates an outline of a clutch finger.

It is important to define key points on the finger for use in calculations throughout the model. On the cannon side of the finger there are two points of interest. The first is the centre of gravity (CG) position. This is where the mass of the finger can be assumed to act, thus is vital for calculation of finger dynamics. The second is the position of the finger end and hence its status (finger free or not). Figure 3 illustrates how these two points are located. 


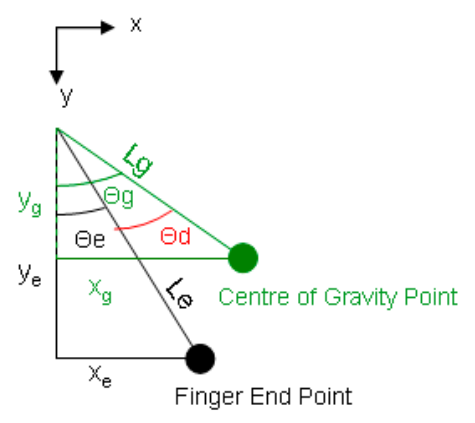

Figure 3: Definition of Key Finger Points

From Figure 3, it can be seen that the relevant positions in $\mathrm{x}$ and $\mathrm{y}$ can defined as follows:

$x_{g}=L_{g} \sin \theta_{g}$

$y_{g}=L_{g} \cos \theta_{g}$

$x_{e}=L_{e} \sin \theta_{e}$

$y_{g}=L_{e} \cos \theta_{e}$

$\theta_{\mathrm{g}}=\theta_{\mathrm{e}}+\theta_{\mathrm{d}}$

The relationships seen above are used within a subsystem in order to create a vector for use in the rest of the clutch module. This vector contains $x$ position, $y$ position and resultant angle for both the CG point and the finger end point. In order to calculate such variables the values of $\theta g, L g, \theta_{e}$ and $L_{e}$ were identified for each individual finger from CAD models.

Optional addition of weights to each finger is incorporated through the use of a vector weighting function as described in Equation 6. The user defines the additional weight added for each finger in the initialisation file.

$\underline{\mathbf{V}}_{\text {new }}=\underline{\mathbf{V}}_{\text {base }} \times m_{\text {finger }}+\underline{\mathbf{V}}_{\text {weight }} \times m_{\text {add }} /\left(m_{\text {finger }}+m_{\text {add }}\right)$

Finger Position and Status Determination - To calculate finger moment (and subsequent clamp load), the position of the finger must first be calculated. The motion of the finger is always restrained by either the cannon or the pressure plate. The initial pressure plate position is assumed to be zero and decreases (becomes more negative) due to wear in the clutch pack during the run. Therefore inputs to the finger position calculator functionality are cannon position and wear. The finger angle for bearing restraint is calculated according to Equation 7.

$\theta e_{\text {not tree }}=\sin ^{-1}\left(x_{c} / L_{e}\right)$

The finger angle for pressure plate restraint is then calculated according to Equation 8. The expression takes account not only of finger geometry but also wear in the clutch pack that leads to a larger pressure plate restraint angle. Note that $\theta r$ is finger tip angle for which the finger would be released if no wear is present (measured from finger geometry). Figure 4 illustrates the geometry of finger interactions with the pressure plate and release bearing.

$\theta e_{\text {free }}=\theta_{r}+\sin ^{-1}\left(x_{w} / y_{f}\right)$

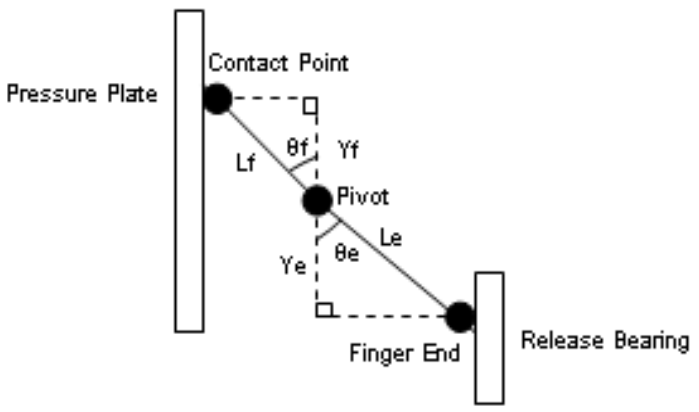

Figure 4: Geometry of Finger Interactions with Pressure Plate and Release Bearing

If the calculated bearing restraint angle is larger than or equal to the pressure plate restraint angle, then the finger must be restrained by the pressure plate. Hence the finger is said to be free and can exert clamp load on the clutch pack. This logic is performed to find the finger angle and whether the finger is free or not and then the finger angle is converted to an angle for the CG position using the value of $\theta_{d}$ for the finger in question. This is then used in the rest of the model. A one way switch is used for finger status, to prevent oscillatory switching at the point of status change.

Finger Centrifugal Force Calculation - Figure 2 also illustrates the rotation of the finger assembly. From this the centrifugal force acting upon each finger can be calculated as:

$\mathrm{F}=\mathrm{mr} \omega^{2}$

The moment acting on the cannon side of the finger can then be calculated as:

$\mathrm{M}=\mathrm{x}_{\mathrm{g}} \mathrm{mr} \omega^{2}$

Clamp Load Calculation - The clamp load caused by a released finger can be determined by equating the moments on both sides of the finger. In order to do this the angle of the contact area on the pressure plate finger side is calculated according to Equation 11.

$\theta_{\mathrm{f}}=\theta \mathrm{e}_{\text {free }}-\theta_{\mathrm{r}}$

Due to the radius on the end of the contact area on the pressure side of the finger, an adjustment is made to the 
value of the length from pivot to contact point according to the value of the contact area angle, as in Equation 12.

$L f_{\text {final }}=L f_{\text {base }}+\left(\theta f / 45^{\circ}\right) \times \Delta L f_{\theta 45}$

Hence the moment on the cannon side of the finger is divided by the perpendicular distance from the contact point to the pivot (on the pressure side of the finger) to find the force clamp load applied to the pressure plate. The perpendicular distance is found using simple trigonometry as seen from Figure 4.

The clamp loads for all 18 fingers are summed. The summed value has the load caused by the pre-load springs subtracted from it to gain the final clamp load value.

Cannon Force Calculation - The cannon force is that imparted onto the release bearing by non-free fingers experiencing centrifugal force. This force is found by dividing the moment on the cannon side by the perpendicular distance from the pivot. This distance is $y_{e}$ (Figure 4). The total cannon force is found by summing the individual contributions from each finger.

From the total force acting upon the cannon, the pressure exerted in the hydraulic fluid can be calculated as:

$P=f / A$

To calculate volume flow velocity through the cannon jets caused by the pressure within the hydraulic fluid, the equivalent hydraulic head must be calculated (Equation 14). Hence volume flow velocity is given by Equation 15.

$\mathrm{H}=\mathrm{P} /(\rho g)$

$Q=A \sqrt{ }(2 g h) k$

The volume flow velocity is found when each jet is active and then the total flow velocity is found and integrated with respect to time in order to find the volume of fluid removed from the hydraulic chamber.

Equation 16 shows how cannon stroke is found from the volume removed from the chamber. Equation 17 yields cannon position from stroke.

Stroke $=\left(V_{\max }-V_{\text {out }}\right) / A_{\text {chamber }}$

$\mathrm{x}_{\mathrm{c}}=\left(\right.$ stroke $_{\max }-$ stroke $)+\mathrm{x}_{\mathrm{c} \text { initial }}$

The initial cannon position is determined by the user in the initialisation file; the user specifies a finger that will be released as soon as the cannon first moves and the initialisation file calculates the bearing position required for the finger angle to be equal to that required for finger release from the cannon.
TEMPERATURE, WORK AND WEAR SUBSYSTEM This subsystem has three functions. Firstly work done within the clutch pack is calculated as:

$W f=\int \mu(t) \omega(t) P(t) d t$

Friction work is then used to estimate clutch flash temperature. Two different cases are analysed. Firstly the internal plates are assumed to be a small thermal mass with poor conduction. This occurs because the steel floaters are insulated on both sides by friction material. Temperature estimation therefore conforms to Equation 19 [2].

$\mathrm{T}_{\mathrm{f}}=\mathrm{nW}_{\mathrm{f}} /(\rho \mathrm{cL})$

The second case applies to the end plates (Equation 20). Infinite thermal resistance is assumed for the friction material such that all the heat that is generated flows though a low resistance steel surface (the flywheel at one end and the pressure plate at the other) to a constant temperature boundary. Uniform heat flux and zero initial temperature are also assumed [3].

$\mathrm{Tf}=\mathrm{FYWf} /[\sqrt{ }(\rho c \lambda t f)]$

The average flash plate temperature is subsequently used as an input for wear estimation. In addition the friction work is used as the other input. Wear is calculated by multiplying the work by a wear coefficient. Since wear varies with temperature, a lookup table is employed to allow for this effect. Separate coefficients are employed for discs and floaters.

CLUTCH TORQUE SUBSYSTEM - From the inputs of clamp load, clutch sliding speed and average clutch temperature, clutch torque is calculated according to Equation 21. A two dimensional lookup table allows for variation of clutch $\mu$ with temperature and sliding speed.

$\mathrm{T}=\mathrm{n \mu N}[($ Router + Rinner $) / 2]$

\section{TYRE MODEL DEVELOPMENT}

Inputs to the tyre model subsystem are vehicle velocity and wheel angular velocity. The outputs are vehicle drive force and driven wheel reaction torque. Pacejka's magic formula is used to calculate tyre $\mu$ as a function of wheel slip (Equation 22) and calculation of driven wheel drag is carried out according to Equation 23.

$\mathrm{y}=\mathrm{D} \sin [\mathrm{C} \arctan \{\mathrm{Bx}-\mathrm{E}(\mathrm{Bx}-\arctan \mathrm{Bx})\}]$

Driven wheel drag $=M g\left(A_{d}+B_{d} v\right)$

VERTICAL LOADS MODEL - The three most significant contributors to vertical load applied to each tyre are:

- Static weight

- Weight transfer occurring under traction

- Downforce caused by the rear wing 
Equation 24 models both static weight and weight transfer occurring under traction.

$\mathrm{Wr}=(\mathrm{Wa} / \mathrm{Lw})+\left(\mathrm{F}_{\mathrm{x}} \mathrm{h} / \mathrm{Lw}\right)$

Aerodynamic loads are modelled according to Equation 25.

$\mathrm{Fz}=0.5 \mathrm{Cdf} \mathrm{v}^{2} \mathrm{~A}_{\text {wingplan }}$

Flow velocity is assumed to be the same as the vehicle velocity. The value of Cdf may vary according to vehicle speed. This occurs because at high flow velocities it is thought wing supporting struts deform due to the large forces present, thus altering angle of wing attack. It is also possible that due to the large flow velocities, the wing may stall, leading to a reduction in downforce. This effect is included through the use of a one dimensional lookup table.

All three vertical loads are summed to find total vertical load. Total vertical load is multiplied by the tyre $\mu$ to gain overall drive force.

TYRE SQUAT AND GROWTH MODELLING - The squat behaviour occurs due to wind up within the tyre sidewall. Hence the sidewall is modelled through the implementation of an additional spring-damper between the wheel and the tyres. This allows wind up to occur at the start of the run. Hence rolling radius squat is modelled as in Equation 26.

$\mathrm{L}_{\text {squat }}=\left(\theta_{\text {wheel }}-\theta_{\text {sidewall }}\right) \mathrm{K}_{\text {tyre torsion }}$

In addition, tyre growth is modelled. This occurs due to centrifugal force generated at high rotational speeds. Hence from the wheel angular velocity, the centrifugal force generated is calculated according to Equation 9. Displacement of the tyre wall is thus calculated according to Equation 27.

$\mathrm{L}_{\text {growth }}=\left(\mathrm{f}_{\text {vertical }}-\mathrm{f}_{\text {centrifugal }}\right) / \mathrm{K}_{\text {tyre radial }}$

Rolling radius is then given by Equation 28 .

$r_{\text {final }}=r_{\text {static }}+L_{\text {growth }}-L_{\text {squat }}$

\section{RESULTS}

Predictions provided by the simulation are compared to actual data obtained from the Lex Joon Racing Dragster for the same set up parameters in order to assess the accuracy of the model. It should be noted that the actual run was performed at Santa Pod raceway in the UK and the data is valid until 4.4 seconds, after which the driver removed his foot from the pedal. Figure 5 shows the engine and axle behaviour for both simulation and actual data.

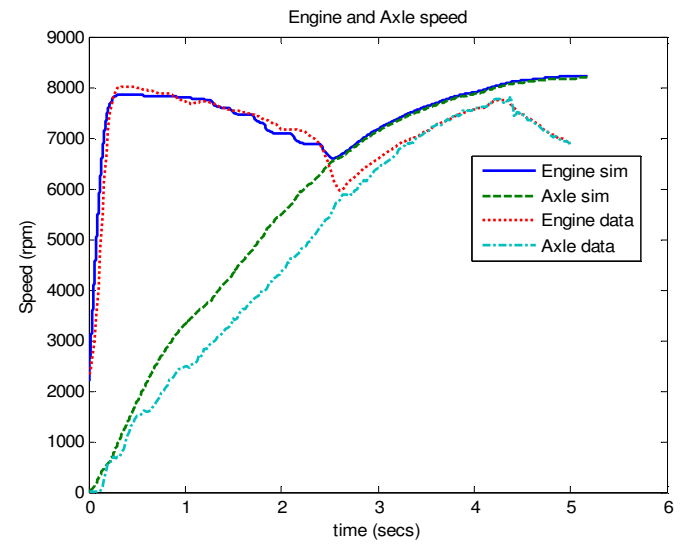

Figure 5: Engine and Axle behaviour

Although the simulation is not completely accurate, similar trends can be seen between simulated and actual data. In particular engine speed prediction is good in the first 2 seconds. Predicted axle output speed seems too high in this region. Both the simulation and actual data show the commencement of clutch lock up at approximately 2.6 seconds; however the drop seen in actual engine speed is not predicted by the simulation. This suggests that either the clutch $\mu$ parameters or the clutch wear parameters (which affects the spread of fingers being released) are not correct. After clutch lock up, the rate at which the synchronised engine and axle speeds increase is similar for both the predictions and actual data.

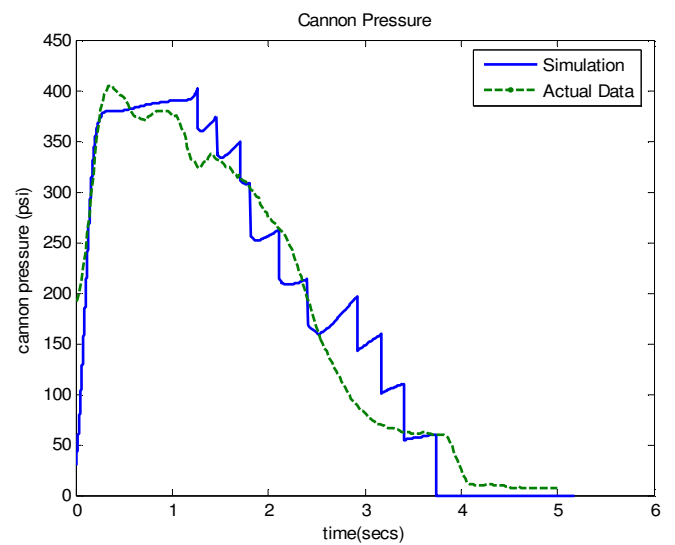

Figure 6: Cannon Pressure Behaviour

Figure 6 shows how the cannon pressure data compares with the predicted behaviour. It can be seen that the simulation shows reasonable predictions of cannon pressure behaviour. The step like behaviour seen in the simulation is caused by the assumption that the finger is either free or not free and hence when the finger becomes free the load is instantly removed from the cannon. In reality there will be a position where the finger is being restrained by both the cannon and the pressure plate, so a tailing off of the load is actually created, leading to the smoother trace seen in the actual data. 
Also it seems possible that the position of the sensor on the dragster could lead to some damping of cannon pressure as it is recorded.

In addition to parameters associated with the clutch system, it is also important to analyse other vehicle behaviours. Figure 7 shows the wheel and vehicle speeds for both the simulation and actual vehicle behaviour. The model predicts the vehicle and wheel speeds accurately for some of the run. In particular the prediction is very good within the region $0-2.5$ seconds. The difference between the wheel and vehicle speeds is indicative of wheel slip including the effects of tyre sidewall wind up, and the prediction of this behaviour is good. After approximately 2.5 seconds, the amount of wheel slip (indicated by the difference in vehicle and wheel speed) predicted is still good, although the magnitude of wheel and vehicle speeds has fallen with respect to the actual data.

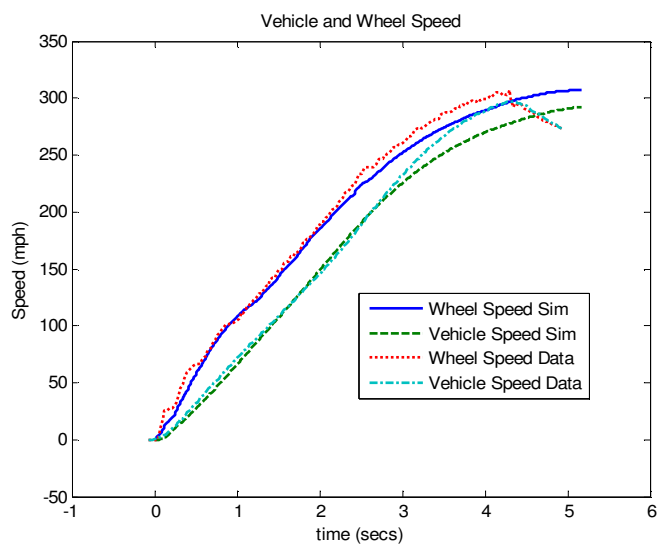

Figure 7: Wheel and Vehicle Speed Behaviour

This discrepancy is possibly due to the fact that the drag parameters are not correct at higher speeds. In the tuning of the model it has been assumed that vehicle drag and down force coefficients do not vary, however due to the large vehicle speeds involved it is possible that these will vary due to aerodynamic separation or deformation of the wing struts. The discrepancy could also be due to slight inaccuracies in the mass and moment of inertia parameters. In particular the vertical loads model and the vehicle mass parameters do not take account of the fuel consumed during the course of a run. A further reason for inaccuracies could be that the torque produced by the engine at a given engine speed will not be constant during the run. This occurs because engine parameters of ignition timing, amount of fuel injected and boost pressure are varied throughout the run. Although the effects of changing ignition timing are included, the effect of changes is probably larger for an engine running on nitro methane than for a typical gasoline engine as was used in deciding the parameters for this effect. In addition the effect of changing fuelling and boost pressure will have very large effects that are not currently included in any capacity.

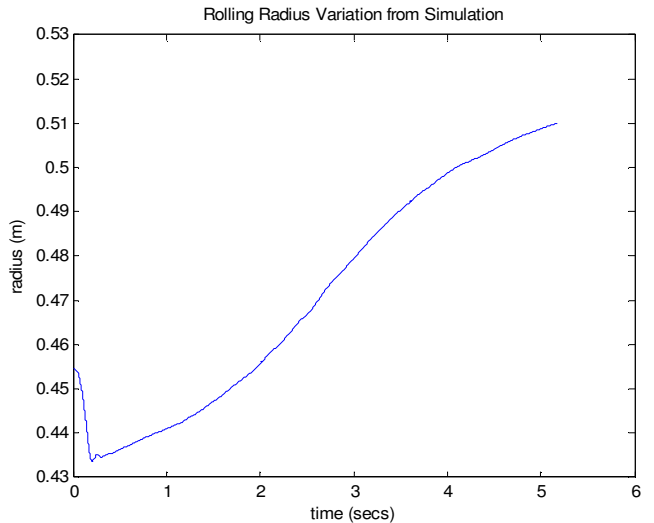

Figure 8: Rolling Radius Variation from Simulation

Figure 8 shows how the rolling radius predicted by the simulation varies. We can see squat and growth behaviour with appropriate magnitudes. This result can only be validated once additional - e.g. laser measurements have been taken from the vehicle during a run.

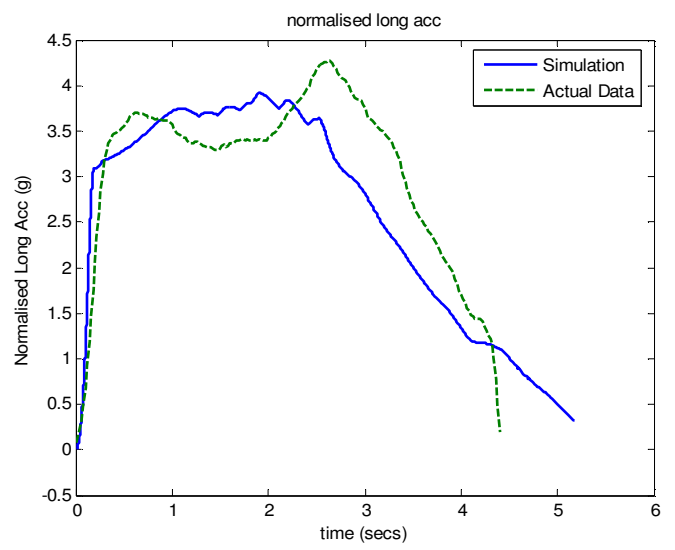

Figure 9: Vehicle Acceleration Behaviour

Finally Figure 9 shows longitudinal acceleration. Although the shape of the acceleration predicted is not identical to the actual vehicle data, the magnitudes at most points seem to be similar. The peak seen at the start of the run for the actual data is predicted slightly later in the simulation. The peak seen at around 2.6 seconds in the actual data is not predicted in the simulation. This is probably due to the smoother clutch lockup predicted by the model. However both plots show a drop off in acceleration after 2.6 seconds and the shape seen during this drop off period is similar.

\section{CONCLUSION}

A suitable basis for top fuel dragster powertrain modelling has been produced. This initial model can be further developed to produce a powerful tuning tool. The overall model structure is in place and requires minimal development. Hence most of the additional development 
work will be further tuning of model parameters. However the results presented here show that significant further tuning work is required in order to produce accurate simulation results.

In terms of the clutch modelling, it can be concluded that a model has been developed that describes the physical processes occurring within the clutch during its operation. This model has functionality for the user to be able to alter the mass added to primary and secondary fingers, the cannon jet opening times, the cannon jet sizes, the initial cannon position and the number of half turns applied to the preload springs. Hence all clutch tuning functionality available in reality is available within the model.

In addition it can be concluded that the clutch model has been incorporated into an overall powertrain model. This overall model accurately reflects the layout of a top fuel dragster powertrain. It has functionality for aspects of vehicle behaviour that are not seen for road vehicles. This includes the rolling radius squat and growth model and vertical loads modelling.

The model shows some accuracy when compared to actual data, where discrepancies have been found possible reasons have been identified and such issues can be addressed when further work is carried out.

Further work could include a function to apply smoothing to the introduction of each finger's clamp load. An aerodynamic study of the vehicle including vehicle drag and rear wing downforce measurements would improve the tuning of the vehicle parameters. The effect of fuelling and boost changes occurring during a run should also be included in the model. Direct measurements of clutch $\mu$ and wear parameters would ensure more accurate simulations. In addition direct measurement of tyre rolling radius during a run would facilitate model improvement.

\section{ACKNOWLEDGEMENTS}

The authors would like to thank Lex Joon and Gerda Joon-Dijkstra from Lex Joon Racing for the opportunity to carry out this work. Also we would like to thank Don Jackson from Don Jackson Engineering (DJE) for his help and advice throughout.

\section{REFERENCES}

1. Bear, J., Dynamics of Fluids in Porous Media, 1972, Dover, ISBN 0486656756

2. Cameron, T. McCombs, S. et al, Flash Temperatures in Clutches, 2005, Society of Automotive Engineers, SAE Paper No 2005-01-3890

3. Benham, P., Crawford, R., and Armstrong, C., Mechanics of Engineering Materials, Second Edition, 1996, Longman, ISBN 0582251648

4. Heywood, J.B., Internal Combustion Engine Fundamentals, 1998, McGraw-Hill, ISBN 007 1004998

\section{CONTACT}

Thomas Stone email address: t_stone@btinternet.com 
DEFINITIONS, ACRONYMS, ABBREVIATIONS

$\underline{\mathbf{V}}$ : mass centre vector

$\mathbf{x}$ : x position $(\mathrm{m})$

y: y position (m)

z: z position (m)

m: mass $(\mathrm{kg})$

$\theta$ : angle (rad)

M: Moment (Nm)

f: Force $(\mathrm{N})$

d: Distance $(\mathrm{m})$

P: Pressure $\left(\mathrm{Nm}^{-2}\right)$

A: Area $\left(m^{2}\right)$

h: hydraulic head $(\mathrm{m})$

p: Density $\left(\mathrm{kgm}^{-3}\right)$

g: Gravitational Constant $\left(\mathrm{ms}^{-2}\right)$

Q: Volume flow rate $\left(\mathrm{m}^{3} \mathrm{~s}^{-1}\right)$

k: Discharge coefficient

Wf: Friction Work (Joules)

$\boldsymbol{\mu}$ : Coefficient of Friction

$\boldsymbol{\omega}$ : Sliding Speed ( $\left.\mathrm{rad} \mathrm{sec}{ }^{-1}\right)$

t: Time (secs)
$\mathbf{T}_{\mathbf{f}}$ : Flash Temperature $(\mathrm{K})$

n: Number of Friction Surfaces

c: Specific Heat $\left(\mathrm{J} \mathrm{kg}^{-1} \mathrm{~K}^{-1}\right)$

L: Length or thickness $(\mathrm{m})$

T: Torque $(\mathrm{Nm})$

$\mathbf{N}$ : Clamp load $(\mathrm{N})$

r: radius $(m)$

$\mathbf{A}_{\mathbf{d}}$ : Tyre Rolling Resistance Coefficient

B $_{\mathrm{d}}$ : Tyre Rolling Resistance Velocity Coefficient

Cd: Coefficient of Drag

$\mathbf{v}:$ Velocity $\left(\mathrm{ms}^{-1}\right)$

W: Weight $(\mathrm{N})$

a: Distance from front axle to location of centre of gravity (m)

$\mathbf{L}_{\mathrm{w}}:$ Wheelbase $(\mathrm{m})$

$\mathbf{F}_{\mathbf{x}}$ : Tractive Force $(\mathrm{N})$

$\mathbf{h}$ : height of $\mathrm{C}$ of $\mathrm{G}$ above road $(\mathrm{m})$

$\mathbf{C}_{\mathrm{df}}$ : Coefficient of downforce of wing

$\mathrm{J}$ : Moment of Inertia $\left(\mathrm{kg} \mathrm{m}^{2}\right)$

V: Volume $\left(\mathrm{m}^{3}\right)$

$\mathbf{K}$ : Tyre stiffness $\left(\mathrm{Nm}^{-1}\right.$ or $\left.\mathrm{Nrad}^{-1}\right)$ 